\title{
Serum tumour markers in malignant mesothelioma
}

\begin{abstract}
Malignant mesothelioma is a rare malignancy of the body cavities with dismal prognosis. It has been a diagnostic dilemma for years with many clinical and pathological mimics. Discovery of a reliable tumour marker will definitely be of value in screening individuals with a history of asbestos exposure, diagnosis, treatment and follow up of malignant mesothelioma. Many tumour markers have been studied and speculatively associated with the malignant mesothelioma, but much still needs to be proven.
\end{abstract}

Key words: Malignant mesothelioma, tumour markers

Malignant mesothelioma is a highly aggressive tumour with an incidence progressively increasing with time. The tumour originates from cells lining the serosal cavities, the most common location being the pleura. It also arises from the peritoneum, pericardium and tunica vaginalis. Asbestos exposure is the prime factor implicated in the pathogenesis of the tumour but few cases have also been associated with exposure to ionising radiation and viral infections. Asbestos occurs predominantly in two forms, the serpentines and the amphiboles, latter implicated in causation of the disease more frequently, owing to its more rigid structure, which causes considerable damage to the mesothelial cells.

Malignant mesothelioma has got a grim prognosis, attributable to the late detection of the disease. Moreover the screening of individuals exposed to asbestos is very difficult because of the extremely long latency period, ranging from 10 to 30 years. The diagnosis of malignant mesothelioma is based on combination of clinical history, radiological appearances and histology. Despite all these modalities, diagnosis remains a challenging task to medical profession as symptoms, imaging and the histological features often mimics other pathologies. Fair numbers of tumour markers are under research with few emerging as promising ones. A reliable tumour marker will simplify and fasten the process of diagnosing malignant mesothelioma, enabling a larger number of patients to be diagnosed in the initial stages and undertake more successful treatment options.

\section{MESOTHELIN PEPTIDE}

Mesothelin is a glycophosphatidylinositol anchored glycoprotein, which has function of cell to cell adhesion. It is a marker of normal mesothelial cells and is over expressed in malignant mesothelioma. However what leads to the release of these peptides from the cell surface to the blood stream is still under speculation. Some suggest a mutation in the gene encoding for the mesothelin protein, leading to release of the protein into the blood stream while others suggest a proteolytic cleavage and release of the soluble fragment of the protein into the circulation.

Robinson et $a l_{r}^{[1]}$ in his study on mesothelin family of proteins, found that patients with malignant mesothelioma had a higher levels of serum mesothelin related peptide (SMR) than the healthy controls (both asbestos exposed and non exposed). Patients with lung adenocarcinoma, metastatic pleural tumours and nonmesotheliomatous pleural effusions did not show raised levels. The data established $84 \%$ sensitivity and $100 \%$ specificity of serum SMR assay in the diagnosis of malignant mesothelioma. The efficacy of serum SMR assay was further substantiated by Scherpereel et al. ${ }^{[2]}$ Raised levels of SMR were found in patients with malignant mesothelioma compared to patients with metastatic tumours to the pleura and benign pleural lesions following exposure to asbestos. SMR assay was also proven to be of value in further studies $^{[3]}$ and levels were found to mirror the tumour bulk and thus can
Pallavi Rao, Srikanth Narayanaswamy Derriford Hospital, Plymouth, United Kingdom

For correspondence: Dr. Pallavi Rao, 28, Catsketon Close, Plymouth PL3 5AE, Plymouth, United Kingdom. E-mail: drpallavirao@gmail.com

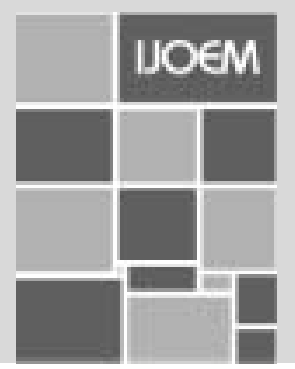


be used for monitoring the course of the disease and to screen patients who are at risk. The levels of SMR may correlate with the histological subtype of the tumour with the highest levels found in the epithelioid subtype. ${ }^{[2]}$ Hassan et al in his study on patients with mesothelioma demonstrated a decrease in the levels of serum mesothelin after surgical treatment suggesting that it can be used to monitor the treatment response. ${ }^{[4]}$

Apart from the usefulness of serum SMR assay in diagnosing mesothelioma, it has also been found to be a useful marker to follow patients who are at risk. People who had an asbestos exposure history and significantly raised SMR levels were followed and found to develop malignant mesothelioma in due course of time. These patients were found to have raised levels one to 4 years before the symptom development. ${ }^{[5]}$ Hence a cautious approach in people showing a raised level is advisable as this may signify a hidden focus of malignancy not detectable by routine methods. ${ }^{[2]}$ It has also been suggested by workers that fluctuations in the serum SMR levels over time may also point towards a developing malignant mesothelioma and can be a very useful approach to follow patients with a history of asbestos exposure. ${ }^{[1]}$ Thus it can be said that it would be a safer approach to investigate at risk people with a raised SMR levels, with more aggressive techniques to rule out a developing malignancy or a very small focus of already developed malignancy, which can definitely add a ray of light to the existing prognosis of patients with malignant mesothelioma.

\section{OSTEOPONTIN}

Osteopontin also known as bone sialoprotein is a glycoprotein considered to be a multifunctional cytokine found in the osseous tissues, brain, kidney, chondrocytes and many other tissues. It is overexpressed in a variety of cancers including malignant mesothelioma.

Studies reveal that serum osteopontin levels were significantly higher in patients with malignant mesothelioma compared to healthy controls with a history of asbestos exposure.$^{[6]}$ However there was no significant difference in the levels of osteopontin among patients with different stages of disease signifying that it did not correlate well with the bulk of the disease. The levels also did not differ in different histological subtypes of the disease. Osteopontin levels have also been found to be elevated in patients with lung, ovarian, colorectal, gastric and pancreatic carcinomas, which can be a drawback to use this marker as a diagnostic marker independently, although it can be of value when used in combination with the existing diagnostic modalities owing to the fact that the duration of the disease and extent of radiological abnormalities correlated well with the levels of osteopontin. ${ }^{[6]}$
The fact that the levels were significantly raised in patients with malignant mesothelioma compared to healthy people with a history of asbestos exposure can be of immense value in screening of people who are at risk. As asbestos exposure is also implicated in causation of malignancies other than malignant mesothelioma (gastrointestinal, laryngeal and urinary), asbestos workers with raised osteopontin levels and no evidence of malignant mesothelioma should be investigated for other malignancies.

\section{SERUM PDGF}

PDGF is a protein secreted from the alpha granules of the platelets and mesenchymal cells. It regulates cell division and growth and also has a role in migration and angiogenesis. It is overexpressed in many inflammatory conditions, atherosclerosis and malignancies. It exists as a diamer with the isoforms $\mathrm{AA}, \mathrm{AB}$ and $\mathrm{BB}$ and binds to the tyrosine kinase family of receptors and signals tumour cell and stroma proliferation through an autocrine or paracrine mode of action.

The AA isoform is secreted by the fibroblasts, osteoblasts and astrocytes. Macrophages and thyroid carcinoma cells secrete predominantly the $\mathrm{BB}$ isoform. Neurons, renal mesangial cells and mesothelial cells secrete the PDGF-AB isoform.

PDGF AB was found to be significantly higher in patients with mesothelioma compared to their healthy controls (both asbestos exposed and non exposed). ${ }^{[7]}$ However the levels in patients with malignant mesothelioma were not significantly different from patients with non small cell lung carcinomas. The levels showed a correlation with the histological subtype, higher levels seen in epithelioid subtype. A significant correlation was also found between the platelet counts and serum PDGF levels, implicating that a high platelet count at diagnosis may be a negative prognostic factor.

Serum PDGF levels can be used as marker to assess the prognosis in patients diagnosed with malignant mesothelioma.

\section{SERUM HYALURONAN}

Hyaluronan is a glycosaminoglycan distributed widely in the body. It is found in the synovial fluid, connective tissue and vitreous humour of the eye. It is an important constituent of extra cellular matrix and functions in lubrication, cell growth and movement.

Serum and pleural fluid hyalouronan levels have been found to be increased in patients with malignant mesothelioma. It can serve as a potential tumour marker or as a marker of 
progression of the disease. ${ }^{[8]}$ The literature reports the sensitivity and specificity of serum hyalouronan to be $65 \%$ and $85 \%$ respectively as an indicator of disease progression. ${ }^{[9]}$ However, the correlation of the serum levels to pleural fluid levels is still under speculation, with few studies reporting a positive correlation and few reporting the contrary.

Patients undergoing an increase in tumour volume manifest increased levels of hyalouronan in the serum while patients who respond to treatment favourably shows a declining trend in the values. ${ }^{[9]}$ All the more patients with highest levels at initial diagnosis seemed to have the shortest survival. ${ }^{[10]}$ The mean concentration of serum hyalouronan in malignant mesothelioma was five to ten times higher than that of normal controls. ${ }^{[1]}$

A cautious approach is advisable in interpreting the levels of serum hyalouronan as raised levels are also seen in patients with liver cirrhosis and chronic inflammatory conditions ${ }^{[12]}$ and the levels may remain normal in small sized tumours. ${ }^{[9]}$

\section{CYFRA 21-1}

CYFRA 21 is a soluble cytokeratin 19 fragment and can be used as a tumour marker in many cancers. As the mesothelial cells express cytokeratin 19 in a very high concentration, the marker may be of use in the evaluation of the disease. Its value in the initial diagnosis may be limited by its wide spread expression in many cells. However levels of CYFRA 21 in concert with a low level of CEA may hint the diagnosis of malignant mesothelioma, while the reverse point towards an adenocarcinoma of the lung.

Paganuzzi et $a{ }^{133}$ observed a significantly higher level of CYFRA 21 in the serum of patients with malignant mesothelioma in comparison to patients with any benign pulmonary disease. However, the difference in the levels between patients with malignant mesothelioma and lung carcinoma was not statistically significant. It can serve as a marker for follow up of patients with established malignant mesothelioma as it is found to have a significant prognostic value for survival. ${ }^{[14]}$ The sensitivity of serum CYFRA 21 levels for the diagnosis of malignant pleural mesothelioma has been stated to be $40 \%$ in the literature and the change in concentrations of the marker reflected the change in tumour burden. ${ }^{[15]}$

\section{TISSUE POLYPEPTIDE ANTIGEN}

Tissue polypeptide antigen is a growth and proliferative cell marker. It is a polypeptide composed of cytokeratin 8,18 and 19. It is found to be elevated in many cancers including squamous cell carcinoma of the head and neck region, salivary gland neoplasms, thyroid cancer and bladder cancers.
Its role in malignant mesothelioma has been investigated in few studies. It was found to be a useful marker in predicting disease progression and survival. ${ }^{[16]}$ The values of TPA correlated well with the levels of CYFRA $21^{[17]}$ and in fact were more sensitive than CYFRA 21 in predicting the disease progression. ${ }^{[18]}$ It has also been helpful in indicating the response to chemotherapy.

CA-125 CA125 belongs to the carbohydrate antigen group of tumour markers and is frequently elevated in ovarian cancers. Elevated levels may also be seen in cancers of breast, endometrium, lung and in a few benign conditions like endometriosis.

Its significance in malignant mesothelioma of the peritoneum still needs to be studied in detail. It can be helpful in the diagnosis in combination with the computed tomographic appearances ${ }^{[19]}$ and also in follow up of patients undergoing chemotherapy as the serum levels reflect the tumour response. ${ }^{[20]}$

\section{FIBRONECTIN}

Fibronectin is a high molecular weight glycoprotein, which binds to receptors known as integrins. It is involved in the process of wound healing, embryogenesis and blood clotting. It exists in 2 forms, an insoluble form found in extracellular matrix and a soluble form found in the plasma.

Diagnostic accuracy of fibronectin in ascitic fluid was nearly $100 \%$ for values more than 110 micrograms $/ \mathrm{ml}$ in predicting malignancy. ${ }^{[21]}$ Its levels in the urine have been found to be useful in prostate and urinary bladder cancers. Its role as a tumour marker in malignant mesothelioma has been investigated by Emri et al. ${ }^{[22]}$ The levels of fibronectin in the pleural fluid and the plasma were elevated in patients with malignant mesothelioma but were not statistically significant in comparison to tuberculous and other nonmesotheliomatous effusions. Thus measurement of serum fibronectin may not add much to the existing diagnostic aids.

\section{OTHERS}

Few case reports have been published stating the paraneoplastic behaviour of the tumour. G-CSF, ${ }^{[2,24]} \mathrm{HCG}^{[25]}$ was found to be secreted by few of the tumours, manifesting as raised leukocyte levels and bilateral gynecomastia respectively.

\section{CONCLUSION}

Several tumour markers have been studied to diagnose and to follow individuals with malignant mesothelioma. Few tumour markers have surfaced as quiet promising. These will 
certainly add to the existing diagnostic modalities, but further studies are required to substantiate the role of these tumour markers as an independent biomarker in the evaluation of MM. There are no established screening programmes, but these tumour markers may emerge as a cost effective screening tool in the near future, thereby reducing the disease morbidity.

\section{REFERENCES}

1. Robinson BW, Creaney J, Lake R, Nowak A, Musk AW, de Klerk N, et al. Mesothelin-family proteins and diagnosis of mesothelioma. Lancet 2003;362:1612-6.

2. Scherpereel A, Grigoriu B, Conti M, Gey T, Marc Grégoire, Copin MC, et al. Soluble Mesothelin-related Peptides in the Diagnosis of Malignant Pleural Mesothelioma. Am J Respir Crit Care Med 2006;173:1155-60.

3. Robinson BW, Creaney J, Lake R, Nowak A, Musk AW, de Klerk N, et al. Soluble mesothelin-related protein-A blood test for mesothelioma. Lung Cancer 2005;49:S109-11.

4. Hassan R, Remaley AT, Sampson ML, Zhang J, Cox DD, Pingpank J, et al. Detection and quantitation of serum mesothelin, a tumor marker for patients with mesothelioma and ovarian cancer. Clin Cancer Res 2006;12:447-53.

5. Creaney J, Robinson BW. Detection of malignant mesothelioma in asbestos-exposed individuals: The potential role of soluble mesothelinrelated protein. Hematol Oncol Clin North Am 2005;19:1025-40.

6. Pass HI, Lott D, Lonardo F, Harbut M, Liu Z, Tang N, et al. Asbestos exposure, pleural mesothelioma and serum osteopontin levels. N Engl J Med 2005;353:1564-73.

7. Filiberti R, Marroni P, Neri M, Ardizzoni A, Betta PG, Cafferata MA, et al. Serum PDGF-AB in pleural mesothelioma. Tumour Biol 2005;26:221-6.

8. Thylen A, Wallin J, Martensson G. Hyaluronan in serum as an indicator of progressive disease in hyaluronan-producing malignant mesothelioma. Cancer 1999;86:2000-5.

9. Dahl IM, Solheim OP, Erikstein B, Muller E. A longitudinal study of the hyaluronan level in the serum of patients with malignant mesothelioma under treatment. Hyaluronan as an indicator of progressive disease. Cancer 1989;64:68-73.

10. Frebourg T, Lerebours G, Delpech B, Benhamou D, Bertrand P, Maingonnat $\mathrm{C}$, et al. Serum hyaluronate in malignant pleural mesothelioma. Cancer 1987;59:2104-7.

11. Dahl IM, Laurent TC. Concentration of hyaluronan in the serum of untreated cancer patients with special reference to patients with mesothelioma. Cancer 1988;62:326-30.

12. Laurent TC, Laurent UB, Fraser JR. Serum hyaluronan as a disease marker. Ann Med 1996;28:241-53.

13. Paganuzzi M, Onetto M, Marroni P, Filiberti R, Tassara E, Parodi S, et al. Diagnostic value of CYFRA 21-1 tumor marker and CEA in pleural effusion due to mesothelioma. Chest 2001;119:1138-42.

14. Bonfrer JM, Schouwink JH, Korse CM, Baas P. Cyfra 21-1 and TPA as markers in malignant mesothelioma. Anticancer Res 1997;17:29713 .

15. Marukawa M, Hiyama J, Shiota Y, Ono T, Sasaki N, Taniyama K, et al. The usefulness of CYFRA 21-1 in diagnosing and monitoring malignant pleural mesothelioma. Acta Med Okayama 1998;52:11923.

16. Hedman M, Arnberg H, Wernlund J, Riska H, Brodin O. Tissue polypeptide antigen (TPA), hyaluronan and CA 125 as serum markers in malignant mesothelioma. Anticancer Res 2003;23:531-6.

17. Schouwink H, Korse CM, Bonfrer JM, Hart AA, Baas P. Prognostic value of the serum tumour markers Cyfra 21-1 and tissue polypeptide antigen in malignant mesothelioma. Lung Cancer 1999;25:25-32.

18. Nisman B, Barak V, Heching N, Kramer M, Reinus C, Lafair J. Cytokeratin markers in malignant pleural mesothelioma. Cancer Detect Prev 1998;22:416-21.

19. Kebapci M, Vardareli E, Adapinar B, Acikalin M. CT findings and serum ca 125 levels in malignant peritoneal mesothelioma: Report of 11 new cases and review of the literature. Eur Radiol 2003;13:2620-6.

20. Simsek H, Kadayifci A, Okan E. Importance of serum CA 125 levels in malignant peritoneal mesothelioma. Tumour Biol 1996;17:1-4.

21. Siddiqui RA, Kochhar R, Singh V, Rajwanshi A, Goenka MK, Mehta SK. Evaluation of fibronectin as a marker of malignant ascites. J Gastroenterol Hepatol 1992;7:161-4.

22. Emri S, Ustundag Y, Budak T, Karakoca Y, Ozdemir O. Assessment of the value of fibronectin as a tumour marker in malignant pleural mesothelioma. Monaldi Arch Chest Dis 1997;52:335-8.

23. Horio H, Nomori H, Morinaga S, Kikuchi T, Tomonari H, Kuriyama $\mathrm{S}$, et al. Granulocyte colony-stimulating factor-producing primary pericardial mesothelioma. Hum Pathol 1999;30:718-20.

24. Kasuga I, Ishizuka S, Minemura K, Utsumi K, Serizawa H, Ohyashiki K. Malignant pleural mesothelioma produces functional granulocytecolony stimulating factor. Chest 2001;119:981-3.

25. Okamoto H, Matsuno Y, Noguchi M, Morinaga S, Fujioka Y, Tsuchiya $\mathrm{R}$, et al. Malignant pleural mesothelioma producing human chorionic gonadotropin. Report of two cases. Am J Surg Pathol 1992;16:969-74.

Source of Support: Nil, Conflict of Interest: None declared. 